\title{
Perfusion Computed Tomography Study: A Utility Tool in Traumatic Cerebral Contusions
}

\author{
Vernon Velho ${ }^{1}$ Hrushikesh U. Kharosekar ${ }^{1}$ Shonali A. Valsangkar ${ }^{1}$ Jasmit Singh ${ }^{1}$ Pravin Survashe ${ }^{1}$ \\ Sucheta Aher $^{2}$
}

1 Department of Neurosurgery, Sir J. J. Group of Hospitals and Grant Medical College, Mumbai, Maharashtra, India

2 Department of Radiology, Sir J. J. Group of Hospitals and

Grant Medical College, Mumbai, Maharashtra, India

Address for correspondence Hrushikesh U. Kharosekar, MCh, Department of Neurosurgery, 4th Floor, Grant Medical College and Sir J. J. Group of Hospitals, Byculla E, Mumbai, Maharashtra 400008, India (e-mail: hkharosekar@gmail.com).

Indian J Neurotrauma 2016;13:23-30.

\begin{abstract}
Keywords

- traumatic

- perfusion

- contusions

- computed tomography
\end{abstract}

\section{Introduction}

Traumatic brain injury (TBI) is a major health problem in India and worldwide, resulting in significant morbidity, mortality, and disabilities of young and productive group of society. Prompt and proper management of TBI within the golden hour can significantly alter their course, especially within first 48 hours of the injury. Newer neuroimaging techniques can determine the presence and monitor the evolution of injury, thus guiding surgical planning in the acute therapy of TBI. ${ }^{1,2}$ One of the best imaging techniques in the acute phase of head injury is noncontrast computed tomography (CT). It is used for the detection of hemorrhage, parenchymal injury, and skull fractures. It is the most relevant imaging procedure for the detection of lesions eligible for surgical intervention as it is widely accessible and done rapidly even in unstable patients. The overall sensitivity of CT for detecting abnormalities is 65 to $75 \%$. Approximately $20 \%$ of the lesions are often missed on CT scan, more so in early phase of TBI. Advances in neuroimaging techniques allow insight into the path physiologic evolution of traumatic head injury, increasing the sensitivity for detecting cerebral contusions and allowing the development of better prognostic indicators. Perfusion CT (PCT) is a functional imaging technique that makes use of the dynamics of contrast material distribution. 
Table 1 Advantages and disadvantages of CT perfusion study

\begin{tabular}{|c|c|}
\hline Advantages & Disadvantages \\
\hline $\begin{array}{l}\text { - More sensitive compared with plain CT scan } \\
\text { - Shows regional blood flow abnormalities in } \\
\text { patients with head injury also } \\
\text { - Can detect regional blood flow anomalies that } \\
\text { cannot be detected with plain CT scan } \\
\text { - Can detect early contusions that might be } \\
\text { missed on early plain CT brain } \\
\text { - Can be helpful in decision making in bilateral } \\
\text { head injuries } \\
\text { - Can be done in same settings when plain CT scan } \\
\text { is done, not time consuming } \\
\text { - Peripheral ischemic zone (penumbra) can be } \\
\text { detected } \\
\text { - Cost-effective as compared with Xenon CT and } \\
\text { SPECT scan } \\
\text { - Very helpful in evolving contusions }\end{array}$ & $\begin{array}{l}\text { - Extra contrast is given } \\
\text { - Cannot be done in patients with renal } \\
\text { dysfunction } \\
\text { - Limited in deeper lesions and posterior fossa } \\
\text { injury due to bone artifact } \\
\text { - Extra radiation exposure thought within limits }\end{array}$ \\
\hline
\end{tabular}

Abbreviations: CT, computed tomography; SPECT, single-photon emission computerized tomography.

PCT is applicable in the emergency setting and can be easily combined with the noncontrast $\mathrm{CT}^{3}$ This is an advantage of doing CT perfusion compared with magnetic resonance imaging [MRI] perfusion (See - Table 1).

The purpose of this study was to evaluate utility of perfusion study in patients with cerebral contusion in relation to outcome.

\section{Objective}

This study aimed to assess the utility of PCT scan of the brain in patients with head trauma having cerebral contusion on CT scan

\section{Materials and Methods}

This was a prospective study performed over a period of 1 year, May 2012 to May 2013, which included 76 patients of head trauma with CT findings suggestive of cerebral contusions, admitted in our department of neurosurgery at Grant Medical College and Sir J. J. Group of Hospitals, Mumbai. Our study was approved by institutional ethics committee.

As a part of study, all these patients underwent CT of the brain without contrast at admission with perfusion scan (with contrast) at same sitting. The contrast injection was explained to patients' relatives and consent was taken. All patients were initially tested for contrast allergy. Patients who were irritable were sedated by accompanying anesthetist. Repeat CT scan with perfusion scan was done at 48 hours irrespective of management strategy. All admission PCT examinations were well tolerated, with no reported side effects, such as allergic reaction to the contrast material, contrast material extravasation, or nephrotoxicity. Patients with hemodynamic instability and deranged renal function test were excluded from the study.

\section{Patient Data}

Total 76 patients were included in our study. Patients' with all age group were included. Patients with GCS (Glasgow Coma Score) of 3 to 15 were included. All patients had predominantly contusions either on plain CT of the brain or on perfusion scan. Patients predominantly having extradural hematoma (EDH), subdural hematoma (SDH), subarachnoid hemorrhage (SAH), or diffuse axonal injury (DAI) were excluded. Hemodynamically unstable patients, patients with deranged renal function test, and uncooperative patients were excluded. Pediatric patients were not included as they were uncooperative and radiation hazard. On admission complete neurologic examination was done, and GCS was noted at admission. Baseline blood investigations, CT of the spine, and abdominal ultrasound were done as a part of protocol.

Patients with low GCS, contusion $>15 \mathrm{~mL}$ were operated without delay after perfusion scan. Repeat plain CT scan and perfusion scan were done after 48 hours irrespective of surgical or conservative management. Patient outcome was evaluated at discharge with GCS and after 3 and 6 months using Glasgow Outcome Scale (GOS) and activities of daily living (ADL) scale.

\section{Imaging Protocol}

Unenhanced cerebral CT was performed, and approximately 30 , 5-mm-thick transverse sections were acquired with $120 \mathrm{kVp}$ and $200 \mathrm{mAs}$. PCT was performed immediately after unenhanced cerebral CT. PCT consisted of a 32-second series, with 40 gantry rotations performed at a rate of one rotation per second in cine mode during intravenous administration of iodinated contrast material. $18 \mathrm{G}$ intravenous angiocatheter was used for venous access. The acquisition parameters for PCT series were $80 \mathrm{kVp}$ and $200 \mathrm{mAs}$. The scan area was selected at the level of contusions or hypodensity that was assessed on unenhanced scan. PCT scan was initiated 7 seconds after injection of $50 \mathrm{~mL}$ of iohexol at a rate of 4.5 to $5 \mathrm{~mL} / \mathrm{s}$ into an antecubital vein by using a pressure injector (Medrad 
pressure injector). The delay before contrast material reached the brain parenchyma allowed baseline images to be acquired without contrast enhancement. Multidetector row CT enabled the assessment of two adjacent 10 -mm-thick sections.

\section{Perfusion Data Processing}

The reconstructed image data were transmitted to work station. Data were evaluated with 3D perfusion software. The software generates the parameter maps that are in gray scale or color coding. When necessary, acquired volumes were corrected for motion with a $3 \mathrm{D}$ rigid motion correction, based on skull movement. Bone was automatically removed with a contour finder that identified the skull bone and an additional segmentation based on Hounsfield unit (HU) values for identifying brain parenchyma. Observations were made based on perfusion abnormalities, its importance in decision making, and relation to postoperative outcome.

\section{Observations and Results}

There were 76 patients enrolled in our study, most of the patients were young adults, 44 were in 20 to 34 years age group (57\%), and 15 were in 35 to 49 years age group (20\%). Fifty (65\%) patients were males and 25 (35\%) were females. Patients younger than 20 years were not a part of the study as pediatric patients were not cooperative during the procedure (-Tables 2 and $\mathbf{3}$ ).

Out of 76 patients, 47 (61\%) were found to have SAH that was the most common associated finding and 17 (22\%) patients had SDH. Cranial vault fracture was seen in 16 (21\%) patients, and 19 patients had multiple contusions. Among these 19 patients with multiple contusions, contusion with largest size, $>15-\mathrm{mL}$ volume was chosen for perfusion scan. None of these patients were in hypotension as they were excluded from study (See - Table 4).

Out of 40 patients operated immediately, 33 had contusions $>15 \mathrm{~mL}$ and midline shift $>10 \mathrm{~mm}$ on admission CT scan with decreased perfusion whereas 7 had hypodensity on plain scan, midline shift $>10 \mathrm{~mm}$, and significantly decreased perfusion with deteriorating GCS correlating with it (-Table 5 ).

Table 2 Age distribution

\begin{tabular}{|l|l|l|l|l|l|}
\hline Age & $\begin{array}{l}20-34 \\
\mathrm{y}\end{array}$ & $\begin{array}{l}35-49 \\
\mathrm{y}\end{array}$ & $\begin{array}{l}50-64 \\
\mathrm{y}\end{array}$ & $\begin{array}{l}65-79 \\
\mathrm{y}\end{array}$ & $\begin{array}{l}>80 \\
\mathrm{y}\end{array}$ \\
\hline $\begin{array}{l}\text { No. of } \\
\text { patients }\end{array}$ & 44 & 13 & 10 & 7 & 2 \\
\hline Percentage & 57 & 20 & 12 & 8 & 3 \\
\hline
\end{tabular}

Table 3 Sex distribution

\begin{tabular}{|l|l|l|}
\hline Sex & No. & Percentage \\
\hline Male & 50 & 65 \\
\hline Female & 25 & 35 \\
\hline
\end{tabular}

Table 4 Associated injuries

\begin{tabular}{|l|l|l|}
\hline Associated CT finding & No. of patients & Percentage \\
\hline Subdural hematoma & 17 & 22.36 \\
\hline Subarachnoid Hemorrhage & 47 & 61 \\
\hline Extradural hematoma & 5 & 6.5 \\
\hline Intraventricular hemorrhage & 3 & 4 \\
\hline Diffuse axonal injury & 2 & 2.6 \\
\hline Cranial vault \# & 16 & 21 \\
\hline
\end{tabular}

Abbreviation: CT, computed tomography; \# indicates fracture.

Table 5 CT findings in operated group of patients $(n=40)$

\begin{tabular}{|l|l|l|}
\hline CT findings & No. of patients & Percentage \\
\hline $\begin{array}{l}\text { Contusion on plain } \\
\text { CT brain }\end{array}$ & 33 & 80 \\
\hline Decreased perfusion & 40 & 100 \\
\hline
\end{tabular}

Abbreviation: CT, computed tomography.

Thirty-six patients who had contusion volume $<15 \mathrm{~mL}$, midline shift $<5 \mathrm{~mm}$, normal perfusion, and GCS of 13 to 15 were managed conservatively. In these patients plain CT scan and perfusion scan were repeated again at 48 hours. Of the 16 patients who required intervention, all had significant decrease in perfusion compared with only 6 showing increased contusion size and increased midline shift on plain CT scan (- Table 6).

Twenty patients in this group had improved perfusion and no increase in contusion size and were managed conservatively.

Among the 40 operated patients, 24 patients showed improved perfusion after surgery and were back to work at 6-month follow-up. Sixteen patients showed no significant improvement in perfusion, 8 among these patients expired, and rest had significant disability at 6month follow-up. Most of these patients had poor

Table 6 Patients requiring delayed intervention in conservative group $(n=16)$

\begin{tabular}{|l|l|l|}
\hline CT findings & No. of patients & Percentage \\
\hline Increase in size of contusion & 6 & 38 \\
\hline Decreased perfusion & 16 & 100 \\
\hline
\end{tabular}

Abbreviation: $\mathrm{CT}$, computed tomography.

Table 7 Management strategy

\begin{tabular}{|l|l|l|}
\hline Management & No. of patients & Percentage \\
\hline Conservative & 20 & 26 \\
\hline Surgery & 40 & 53 \\
\hline Delayed intervention & 16 & 21 \\
\hline
\end{tabular}



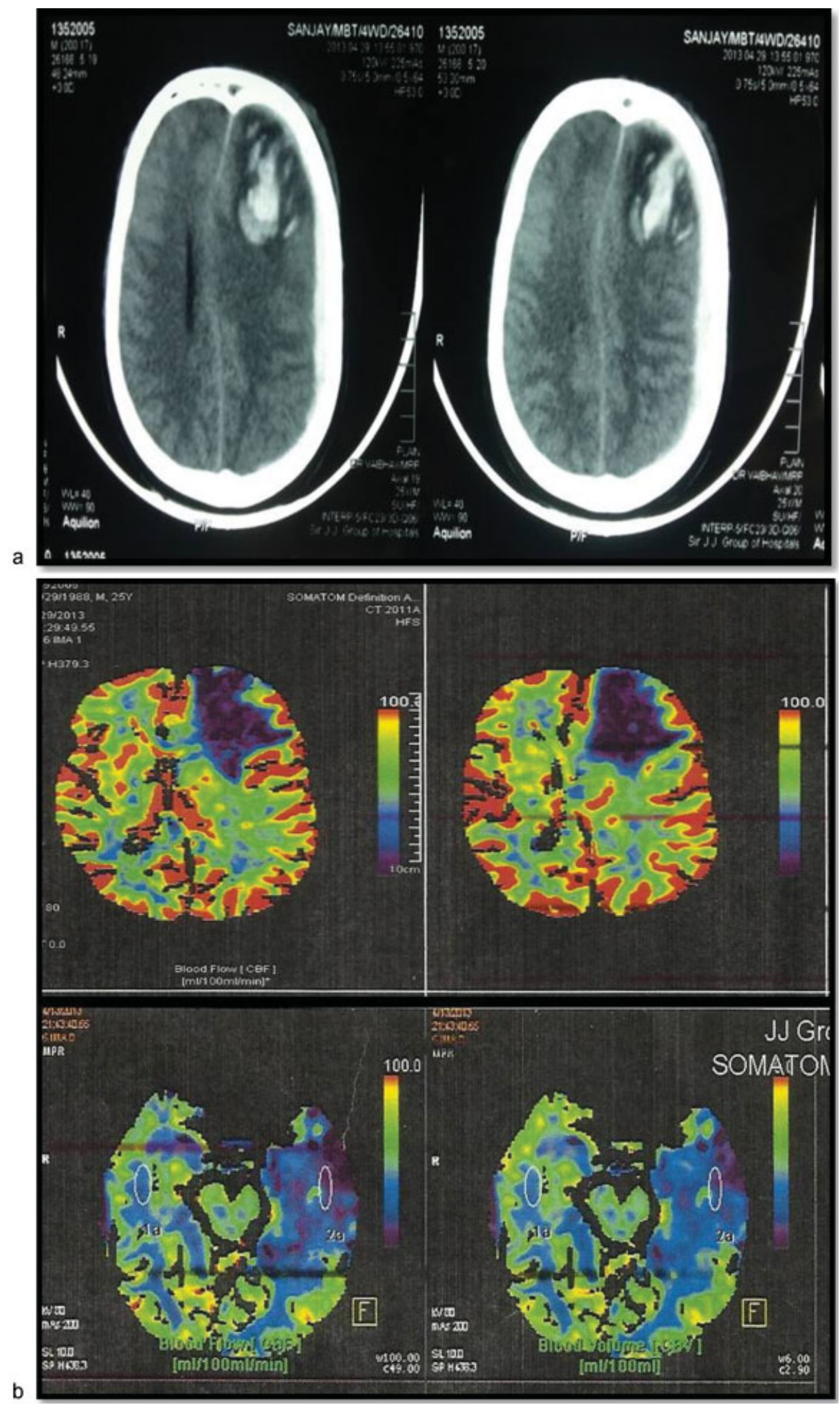

Fig. 1 (a) CT of the brain of a 20-year-old male patient with left frontal contusion at admission. (b) Perfusion scans of the same patient at admission showing significant reduction in perfusion at that site. Also, lower one showing perfusion scan 48 hours after surgery in the same patient showing significant decreased perfusion despite decompression. It correlated well with outcome of the patient as the patient had significant deficits at discharge and was discharged with tracheostomy.

prognostic risk factors such as presentation $>24$ hour after trauma, old age, or significant associated lesion (-Table 7).

\section{Perfusion Abnormality and Its Correlation}

There were 10 patients who were initially conserved with no increase in contusion size on follow-up CT scan but had 
decreased perfusion required surgery and improved after surgery. These patients were operated as decreased perfusion (suggestive of decreased blood flow in penumbra) was considered early sign of evolution of contusion. This prevented deterioration in GCS and helped in improving the outcome in our view. This needs further controlled trials.

Sixteen patients with poor outcome, 8 of whom died, had significant decreased perfusion that did not improve even after surgery.

Patients with improved perfusion or maintained perfusion at admission had good outcome and were back to work at 6 month ( - Figs. 1 -4).

\section{Discussion}

TBI remains a challenge due to its increasing incidence in this era of high-speed vehicles and complex nature of injuries. It can manifest as subdural hematomas, extradural hematomas, cerebral contusions, diffuse axonal injury, and subarachnoid hemorrhage independently or multiple lesions simultaneously. As most of the patients suffering TBI are young adults, saving their life and improving the outcome remain the goal.

CT is a sensitive primary diagnostic tool in the evaluation of patients with acute head injury and plays a critical role in the early detection of intracranial lesions that may require

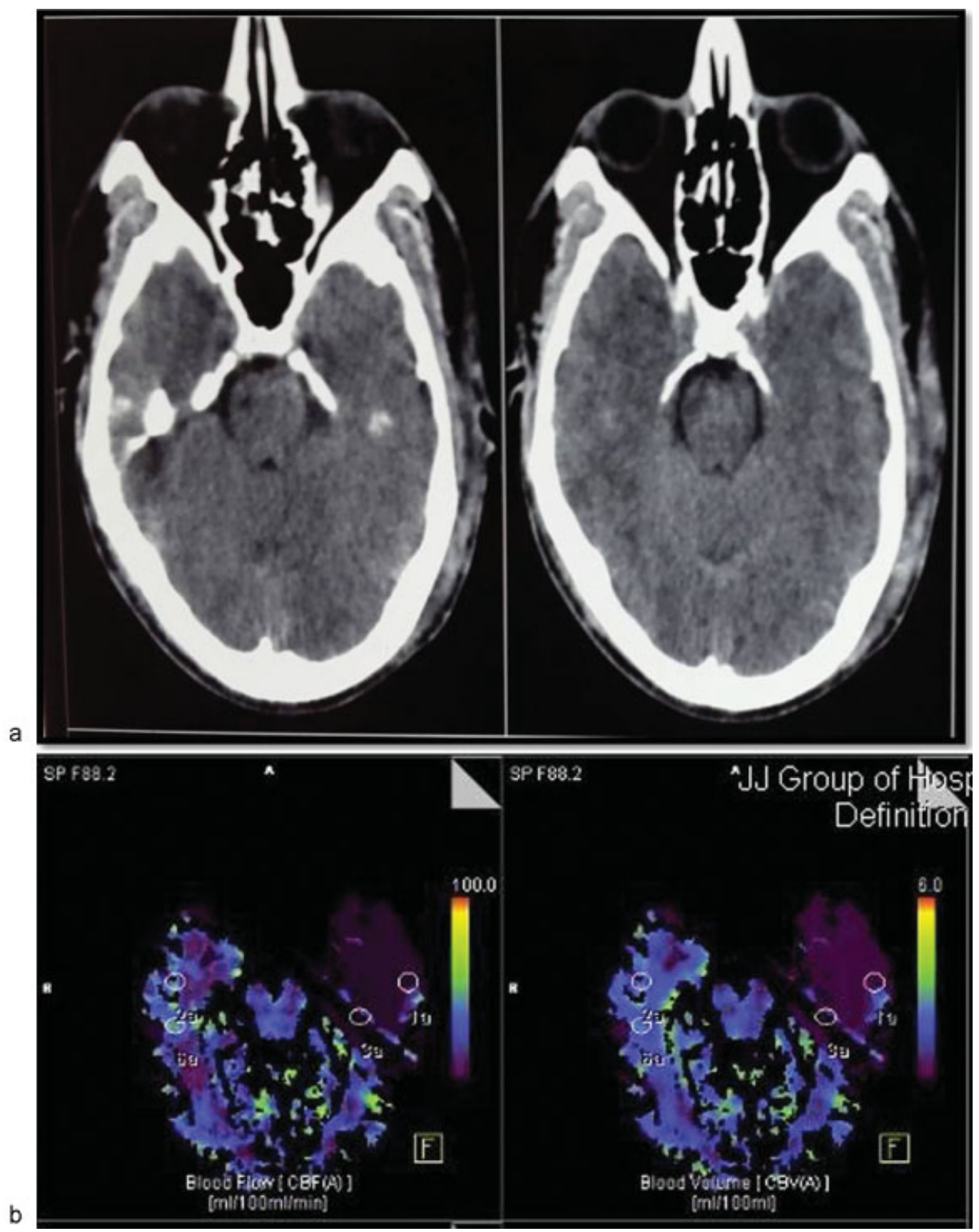

Fig. 2 (a) Plain CT of the brain in an elderly male patient showing only mild edema in left temporal region. (b) Perfusion scan in the same patient showing significantly decreased perfusion in same area compared with surrounding brain parenchyma. (c) Plain CT of the brain of the same patient after 48 hours showing well-developed contusion in same region that was hypoperfused, patient had to be operated for it. (d) Postsurgery perfusion scan of the same patient showing minimal improvement in perfusion; the patient had right-sided hemiparesis and tracheostomy at discharge. 


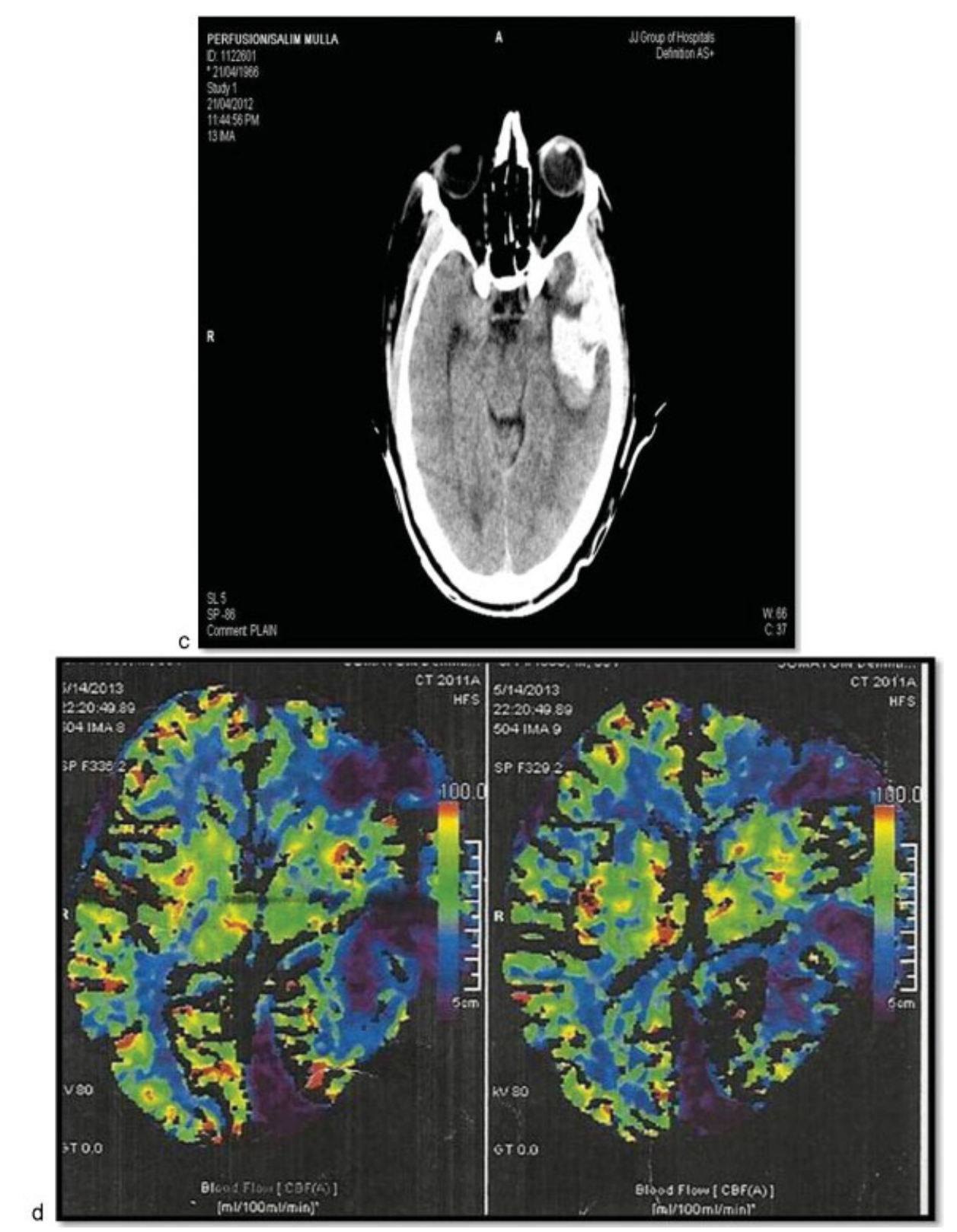

Fig. 2 (Continued)

neurosurgical intervention. Several methods for classifying CT features have been proposed, but unfortunately the prognostic value of such classifications based on the results of admission CT has shown limited value. On the one hand, early CT examinations (those performed within 3 hours of injury) lead to underestimation of the ultimate size of parenchymal lesions. On the other hand, conventional CT does not afford insight into secondary ischemic injuries related to traumatic cerebral edema and intracranial hypertension, which are responsible for about half of all deaths after admission. ${ }^{4}$

PCT can easily be performed as a complement to conventional unenhanced and contrast-enhanced cerebral $\mathrm{CT}$, and does not interfere with other investigations. PCT provides insight into regional brain perfusion alterations due to head trauma, with the major advantage of being able to detect regional heterogeneity. For instance, PCT can quantify the effect of blood collection on the perfusion of the adjacent brain parenchyma. PCT has a higher sensitivity for the diagnosis of cerebral contusions when compared with admission noncontrast cerebral CT. This increased accuracy of PCT to define cerebral contusion may be of critical importance for identification of nonhemorrhagic contusions missed on initial noncontrast CT. In mixed lesions the nonhemorrhagic hypodense component of the cerebral contusion contusions cannot be easily differentiated from perilesional edema, making the delineation of the true extent of the lesion challenging and often inaccurate. In these situations, analysis of PCT parameters within and around the lesion may be particularly useful. Furthermore, cerebral contusions are characterized by their specific capacity to enlarge and turn into large hemorrhagic 

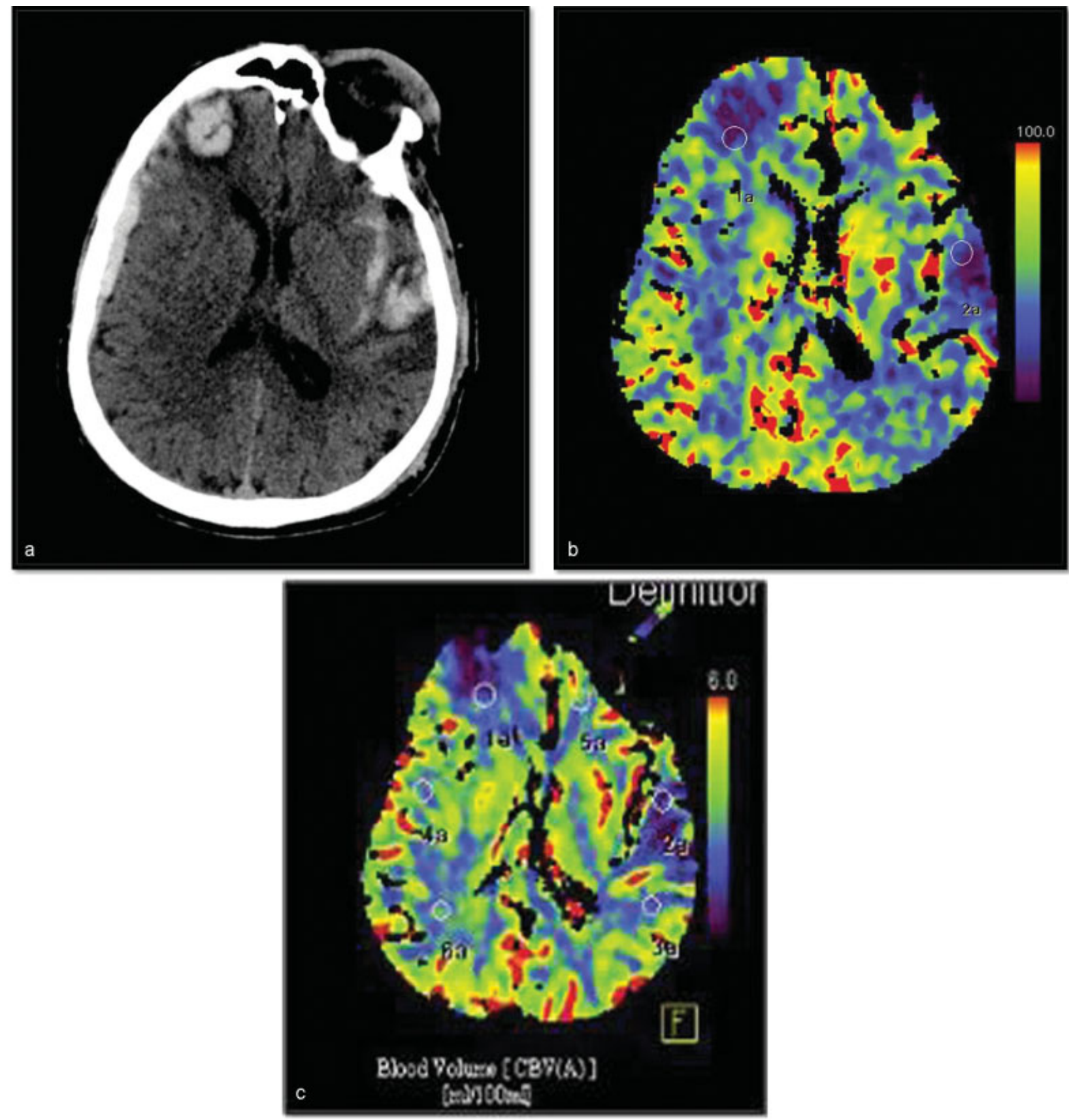

Fig. 3 (a) Plain CT of the brain of a young adult showing right frontal and left temporal contusion. The patient was admitted with GCS of 15/ 15. (b) Perfusion scan of the same patient at admission showing maintained perfusion in surrounding brain parenchyma. The patient was conservatively managed based on these findings. (c) Perfusion scan 48 hours later in the same patient showing improving perfusion; the patient was well preserved and discharged without any deficits.

lesions, and have been shown to represent the most frequent indication for delayed surgical intervention. Accordingly, cerebral contusions represent a dilemma due to associated peripheral viable tissue of critical importance, especially in neurologically eloquent areas. In a recent study, PCT proved to be a useful tool for differentiating necrotic from viable tissue, therefore suggesting its implementation for surgical planning in neurologically eloquent areas. Also, early PCT proved to better correlate with final tissue outcome than noncontrast CT, a fact of importance for identification of patients at high risk of lesion growth. ${ }^{5}$ Patients with maintained perfusion at admission or with improved perfusion had better outcome at 6-month follow-up whereas those with decreased perfusion had poor outcome.

The results of this study demonstrate that PCT provides additional information with respect to focal brain traumatic injuries when compared with conventional CT of the brain. Indeed, PCT had a higher sensitivity for the diagnosis of 


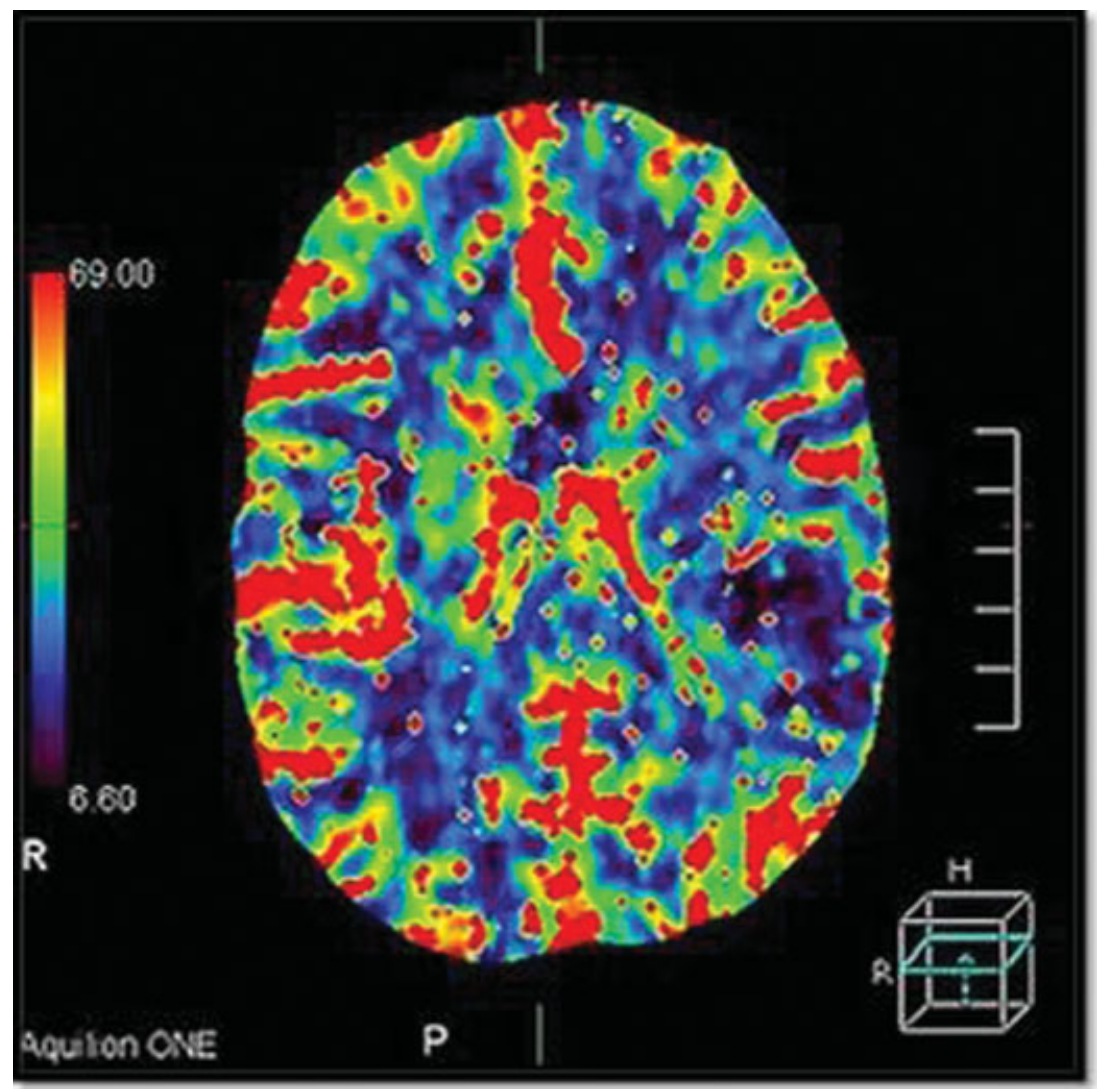

Fig. 4 Normal perfusion scans of the brain.

Table 8 Sensitivity

\begin{tabular}{|l|l|}
\hline $\begin{array}{l}\text { Sensitivity of admission perfusion } \\
\text { CT in detecting contusions: } \\
36 / 38 \times 100=94.7 \%\end{array}$ & $\begin{array}{l}\text { Chi-square }\left(x^{2}\right)-97.6 \\
p \text { value }<0.005 \\
\text { Highly significant }\end{array}$ \\
\hline $\begin{array}{l}\text { Sensitivity of admission } \\
\text { CT in detecting contusions: } \\
24 / 38 \times 100=63.15 \%\end{array}$ & \\
\hline
\end{tabular}

Abbreviation: $\mathrm{CT}$, computed tomography.

cerebral contusions when compared with admission unenhanced cerebral CT, with a sensitivity of $94.7 \%$ versus a sensitivity of $63.15 \%$, respectively ( - Table 8 ). These results are comparable with results published by Wintermark in which comparable sensitivities were $87.5 \%$ and $39.6 \%$, respectively. ${ }^{4}$

\section{Conclusion}

PCT can be performed in patients with severe head trauma, even in the emergency setting. PCT detects regional brain perfusion alterations due to head trauma, with the major advantage of being able to detect regional heterogeneity. Its results show specific patterns that are linked to cerebral edema and intracranial hypertension. PCT offers useful information with respect to functional outcome.

Perfusion scan has its limitations and technical difficulties in certain areas like frontal skull base. The value needs to evaluate with further controlled trials to incorporate it as routine investigation in evaluation of TBI.

\section{References}

1 Gururaj G. Epidemiology of traumatic brain injuries: Indian scenario. Neurol Res 2002;24(1):24-28

2 Lee B, Newberg A. Neuroimaging in traumatic brain imaging. NeuroRx 2005;2(2):372-383

3 Metting Z, Rödiger LA, Stewart RE, Oudkerk M, De Keyser J, van der Naalt J. Perfusion computed tomography in the acute phase of mild head injury: regional dysfunction and prognostic value. Ann Neurol 2009;66(6):809-816

4 Wintermark M, van Melle G, Schnyder P, et al. Admission perfusion $\mathrm{CT}$ : prognostic value in patients with severe head trauma. Radiology 2004;232(1):211-220

5 Wintermark M, Soustiel J-F. Perfusion-CT: A non-invasive tool to monitor regional brain perfusion in traumatic brain injury patients and therapies impacting it. Open Neurosurg J 2010; 3:59-63 